current and future pharmacological interventions of the prostaglandin pathway. The strong suppressive effect on SP in vagus projected areas reveals the importance of CAP in complex brain networks.

\section{A2.8 ENHANCED NEUTROPHIL EXTRACELLULAR TRAP FORMATION IN RHEUMATOID ARTHRITIS PATIENTS IS CORRELATED WITH HIGH LEVELS OF RHEUMATOID FACTOR (RF)}

doi:10.1136/annrheumdis-2013-203215.8

${ }^{1}$ Garyfalia Papadaki, ${ }^{1}$ Christiana Choulaki, 'George Bertsias, ${ }^{2}$ loannis Mitroulis, ${ }^{3}$ Panayotis Verginis, ${ }^{4}$ Argyro Repa, ${ }^{4}$ Amalia Raptopoulou, ${ }^{5}$ Dimitrios Boumpas, 1.4Prodromos Sidiropoulos. 'Laboratory of Autoimmunity and Inflammation, University of Crete, Medical School, Heraklion, Greece; ${ }^{2}$ First Department of Internal Medicine, Democritus University of Thrace, Alexandroupolis, Greece; ${ }^{3}$ Division of Immunobiology, Biomedical Research Foundation Academy of Athens, Athens; ${ }^{4}$ Department of Rheumatology, University of Crete, Medical School, Heraklion, Greece; ${ }^{5}$ Institute of Molecular Biology and Biotechnology, Foundation for Research and Technology, 71300 Heraklion, Greece

Background/Objectives Neutrophils are the most abundant cell type identified in joints from patients with rheumatoid arthritis (RA), with a key role in inflammation and cartilage damage. Activated neutrophils form extracellular traps (NETs) with potent proinflammatory and immunostimulatory activity. Consequently, we sought to assess the role of NET release (NETosis) in RA pathogenesis and whether RA specific autoantibodies (rheumatoid factor $[R F])$ are correlated to this phenomenon.

Materials/Methods Peripheral blood neutrophils were isolated from active RA patients $(n=6)$ (Disease activity score, DAS28 > 5.1) and healthy control subjects $(n=7)$. NET formation from neutrophils, both spontaneous and following incubation with RA serum ( $n=7)$ or synovial fluid $(n=7)$, was assessed by immunofluoresence microscopy, using co-staining with myeloperoxidase and 4',6-Diamidino-2-phenylindole dihydrochloride (DAPI). The percentage of NET releasing cells was determined by examining 200 cells per sample in a double blind fashion. Extracellular DNA content was quantified by fluorescence spectrometry (picogreen) and NET fold increase was calculated based on the extracellular DNA content produced by healthy unstimulated neutrophils.

Results Freshly isolated neutrophils from the peripheral blood of RA patients underwent spontaneous NETosis at higher rates compared to healthy controls $(12 \pm 2.1 \%$ versus $3.2 \pm 0.9 \%, \mathrm{p}<0.05)$. Notably, neutrophils isolated from RA synovial fluid exhibited even higher rates of NETosis. Incubation of healthy neutrophils with RA serum or synovial fluid induced NET release compared to the effect of normal serum $(16 \pm 2.5 \%$ and $9 \pm 1.5 \%$, versus $3.2 \pm 0.7 p<0.005)$. Moreover, quantification of the extracellular DNA content revealed that neutrophils from RF positive RA patients $(n=6)$ exhibited significantly increased spontaneous NET formation compared to RF negative patients $(\mathrm{n}=8)(542 \pm 115.2 \mathrm{ng} / \mathrm{ml}$ versus $199 \pm 52.84 \mathrm{ng} / \mathrm{ml}$, $p=0.008$ ). Treatment of healthy neutrophils with either serum or synovial fluid derived from RF positive RA patients increased NETs compared to cells treated with normal serum (1.45 \pm 0.19 and $4.85 \pm 1.5$ fold increase, $p<0.05)$. Inhibition studies are in progress to address the role of inflammatory cytokines (TNF-a, IL-6, IL-1b) and immune complexes in RA NETosis. Preliminary data show that NET induction in healthy neutrophils upon treatment with RA serum/synovial fluid may be mediated through Fc $\gamma$ receptors.

Conclusions We found that neutrophils from RA patients have enhanced NET formation, driven by soluble factors found in RA sera and synovial fluid, and this is associated with presence of RF. Further studies will address whether NETs are involved in the initiation of adaptive immune responses in humans and in mouse model of arthritis, and whether suppression of NETosis may ameliorate arthritis in RA mouse models.

\section{A2.9 HIGH SERUM-CHOLESTEROL LEVELS BY EITHER LOW DENSITY LIPOPROTEIN RECEPTOR DEFICIENCY OR A CHOLESTEROL-RICH DIET RESULT IN SYNOVIAL ACTIVATION AND OSTEOPHYTE FORMATION DURING EXPERIMENTAL OSTEOARTHRITIS}

doi:10.1136/annrheumdis-2013-203215.9

W de Munter, AW Slöetjes, B Walgreen, MM Helsen, WB van den Berg, PL van Lent. Department of Experimental Rheumatology \& Advanced Therapeutics. Radboud University Nijmegen Medical Centre, The Netherlands

Background and Objectives Atherosclerotic studies show that scavenger receptors on macrophages are capable of taking up oxidised low density lipoprotein (oxLDL), resulting in increased inflammatory properties of the macrophage. Accumulated LDL can be oxidised in an inflammatory milieu such as $\mathrm{OA}$, possibly resulting in oxLDL uptake of synovial macrophages. We investigated whether increased LDL levels lead to more severe OA pathology in experimental induced $O A$

Materials and Methods LDL receptor deficient $\left(\mathrm{LDLr}^{-/}\right)$mice and their wild type (WT) controls received either a cholesterol-rich or control diet for 120 days. Experimental OA was induced by intraarticular injection of collagenase. 36 days after $\mathrm{OA}$ induction, mice were sacrificed and total knee joints and serum were collected. Bone marrow derived cells were differentiated into type two macrophages and pre-incubated with oxLDL for 24 hours and stimulated with S100A8. RNA was analysed for gene expression. Data are depicted as mean \pm SEM.

Results WT mice receiving a normal diet developed moderate cartilage destruction $(6.1 \pm 1.5)$, synovial thickening $(1.4 \pm 0.2)$ and osteophyte formation $\left(32.4 \mu \mathrm{m}^{2} \pm 14.6\right)$. Serum LDL levels were significantly higher in $\mathrm{LDLr}^{-/}$mice compared to WT mice (7.33 $\mathrm{mmol} / \mathrm{L} \pm 0.46$ and $0.54 \mathrm{mmol} / \mathrm{L} \pm 0.04$ respectively; $\mathrm{p}<0.05)$, which was additionally increased by a cholesterol-rich diet $(38.73$ $\mathrm{mmol} / \mathrm{L} \pm 3.11 ; \mathrm{p}<0.0001)$. Despite differences in serum LDL levels, no significant differences between the four groups were found regarding synovial thickening and cartilage destruction. Expression of S100A8 by the synovial lining, however, was increased after receiving a cholesterol-rich diet, suggesting synovial activation. Furthermore, a cholesterol-rich diet increased ApoB accumulation in synovial lining macrophages of LDLr $\%$ mice. Interestingly, at the tibial plateau, $\mathrm{LDLr}^{-1}$ mice showed almost a fourfold increase of osteophyte formation compared to WT mice $\left(206.3 \mu \mathrm{m}^{2} \pm 36.3\right.$; $\mathrm{p}<0.05)$. When receiving a cholesterol-rich diet, osteophyte formation at the lateral side of the tibial plateau in $\mathrm{LDLr}^{-/}$mice further increased from $107.0 \mu \mathrm{m}^{2} \pm 49.3$ to $309.4 \mu \mathrm{m}^{2} \pm 41.7(\mathrm{p}<0.05)$. In vitro stimulation of oxLDL-laden macrophages with S100A8 showed a significant decrease of IL-10 expression and an increase of BMP6 expression compared to macrophages that were not preincubated with oxLDL.

Conclusions Increased serum cholesterol levels by either LDL receptor deficiency or a cholesterol-rich diet increase oxLDL uptake by synovial lining macrophages and synovial activation. In accordance with in vitro data, this synovial activation by oxLDL leads to an inflammatory milieu with increased S100A8 levels, resulting in increased osteophyte formation.

\section{A2.10 INCREASED IMMUNOLOGIC EXPOSURE TO NECROTIC CELL REMNANTS IN PATIENTS WITH PRIMARY SJÖGREN'S SYNDROME OWING TO DEFECTIVE DNASE-I ACTIVITY AND THE PRESENCE OF OPSONIZING IGG AUTOANTIBODIES IN SERUM}

doi:10.1136/annrheumdis-2013-203215.10

Aigli G Vakrakou, George Fragoulis, Menelaos N Manoussakis. Dpt. Pathophysiology, University of Athens, Greece 